\section{Fatores associados à auto-aplicação da insulina nos usuários com diabetes mellitus acompanhados pela Estratégia Saúde da Família}

\author{
Factors associated with insulin self-administration \\ by diabetes mellitus patients in the Family Health \\ Strategy
}

\footnotetext{
${ }^{1}$ Hospital de Clínicas, Universidade Federal do Triângulo Mineiro, Uberaba Brasil.

2 Escola de Enfermagem de Ribeirão Preto

Universidade de São Paulo, Ribeirão Preto, Brasil.

Correspondência

T. S. G. Stacciarin Serviço de Educação em Enfermagem, Hospital de Clínicas, Universidade

Federal do Triângulo Mineiro.

Rua José Pimenta Camargo 332, Uberaba, $M G$

38081-230, Brasil.

thais.stacciarini@terra.com.br
}

\begin{abstract}
This cross-sectional study aimed to compare two groups of patients with diabetes mellitus treated under the Family Health Strategy, with insulin self-administration versus non-self-administration, in relation to socio-demographic variables, perceived difficulties, and the person responsible for the self-application. A total of 269 patients participated, included through simple random sampling, from 37 Family Health Strategy units in the urban area of a municipality in the State of Minas Gerais, Brazil. The self-administration group consisted of 169 individuals (62.8\%), as compared to 100 (37.2\%) in the non-self-administration group. Comparing the two groups, schooling was statistically significant; 45\% of those who did not self-administer reported absence of physical or cognitive difficulties that might prevent them from conducting the procedure, demonstrating the potential for adherence; 90\% reported needing assistance in the insulin administration process at home, and of these, $75 \%$ reported receiving assistance from family members. The Family Health Strategy favors the development of interventions centered on users' needs, encouraging them to adopt and develop self-care skills, and the results of the present study can contribute to the planning of such interventions.
\end{abstract}

Diabetes Mellitus; Insulin; Self Care; Nursing; Family Health
Thaís Santos Guerra Stacciarini

Vanderlei José Haas 2

Ana Emilia Pace 2

\section{Introdução}

Com o objetivo de conseguir um bom controle metabólico entre as pessoas com diabetes mellitus, o tratamento substitutivo com insulina exógena constitui a opção terapêutica mais eficiente, frente à deficiência parcial ou total da secreção de insulina 1,2 .

A terapia com insulina visa a mimetizar, tanto quanto possível, o perfil fisiológico da secreção pancreática de insulina. Dessa forma, múltiplas doses diárias desse hormônio no tecido subcutâneo são necessárias no sentido de proporcionar o controle glicêmico, o qual tem sido demonstrado como condição essencial na prevenção das complicações agudas e crônicas do diabetes mellitus 3 .

Mas para que o controle glicêmico seja efetivo com o tratamento insulinoterápico, é necessário que o usuário com diabetes mellitus aprenda vários aspectos sobre como utilizar a insulina exógena, pois a ação deste medicamento está diretamente relacionada a fatores envolvidos desde a sua aquisição até a aplicação ${ }^{4}$ e que, para alcançar este objetivo, é necessário tempo, prática e educação permanente para o desenvolvimento de confiança e habilidade técnica 5 .

No entanto, estudos têm mostrado que a realidade do cuidado ao usuário com diabetes mellitus continua deficiente, apesar dos avanços do tratamento insulinoterápico nos últimos anos, pois ainda se observa déficit de conhecimento 
em relação aos procedimentos básicos para a aplicação de insulina, tais como: delimitação da região de aplicação, rodízio dos locais de aplicação, conservação da insulina, entre outros 6,7 .

As práticas inadequadas e inseguranças na auto-aplicação da insulina podem interferir no controle metabólico e, conseqüentemente, influenciar a progressão das complicações crônicas do diabetes mellitus ${ }^{8}$. Desse modo, há necessidade de investimentos nessa área, uma vez que erros na administração da insulina poderão ocorrer com maiores freqüências entre aqueles que não auto-aplicam ${ }^{9}$.

Estudos apontam uma freqüência de $52 \%$ a $71 \%$ para a auto-aplicação de insulina entre as pessoas com diabetes mellitus 8,10,11,12. Alguns justificam que a auto-aplicação da insulina reflete o grau de independência, leva ao autocuidado e ao melhor controle metabólico 8,10, e outros atribuem o elevado número de pessoas que não auto-aplicam a falhas no plano educacional 11,12.

Tendo em vista a relevância da produção de novos conhecimentos e o desenvolvimento de novas práticas de saúde consoantes com os princípios e diretrizes do Sistema Único de Saúde (SUS), a Estratégia Saúde da Família aponta como um modelo de reorientação assistencial privilegiado para o desenvolvimento de práticas de promoção para o autocuidado devido às suas características de maior proximidade com a população e ênfase nas ações preventivas e promocionais ${ }^{13}$, permitindo a identificação mais acurada e um melhor acompanhamento dos usuários com diabetes.

Diante do exposto, propôs-se o presente estudo com os objetivos de comparar o grupo de usuários que faz a auto-aplicação de insulina com o que não faz a auto-aplicação, segundo as variáveis sócio-demográficas e clínicas; para o grupo que não faz a auto-aplicação, conhecer as dificuldades percebidas para a auto-aplicação da insulina e verificar quem realiza este procedimento.

\section{Metodologia}

Trata-se de um estudo seccional com abordagem quantitativa, desenvolvido em 37 unidades da Estratégia Saúde da Família da área urbana de um município, referência regional nas áreas da saúde, educação e economia, do Estado de Minas Gerais, Brasil, no período de agosto a outubro de 2006.

A opção pela área urbana foi devido ao fácil acesso dos pesquisadores para a coleta de dados, além da inconsistência dos cadastros relativos à zona rural, os quais não revelavam exatamente a localização dos usuários que utilizam a insulina.

A população-base foi constituída por 781 usuários com diabetes mellitus que fazem uso de insulina, cadastrados e acompanhados pela Estratégia Saúde da Família da área urbana do município onde foi desenvolvido o estudo.

Os critérios de inclusão adotados foram: ter idade igual ou superior a 18 anos; ser cadastrado na Estratégia Saúde da Família; fazer uso da insulina há mais de um ano; e utilizar seringas para a aplicação da insulina.

De acordo com o estabelecimento dos critérios de inclusão, foram excluídos 131 usuários, sendo que 31 deles apresentavam idade inferior a 18 anos, 84 aplicavam a insulina há menos de um ano, 12 foram cadastrados há menos de um ano na Estratégia Saúde da Família e quatro utilizavam a caneta injetora de insulina para a aplicação. Desse modo, a população do estudo foi constituída por 650 usuários que atenderam aos critérios de inclusão previamente estabelecidos neste estudo.

A partir do levantamento dos 650 usuários, elaborou-se uma lista com a finalidade de sortear os usuários distribuídos nas 37 unidades da Estratégia Saúde da Família, empregando-se, por amostragem aleatória simples, o aplicativo SPSS versão 15.0 (SPPS Inc., Chicago, Estados Unidos). Para o cálculo do tamanho amostral considerouse uma prevalência de auto-aplicação de $50 \%$, com uma precisão de 5\%, intervalo de $95 \%$ de confiança e uma perda amostral de $15 \%$.

Dessa forma, a amostra foi constituída por 286 usuários. Durante o estudo, houve perda de 17 pessoas, sendo 11 não encontradas após três visitas domiciliares, uma hospitalizada, três óbitos e duas recusas em participar do estudo.

Após a definição das variáveis foram elaborados dois instrumentos de coleta de dados, considerando ampla revisão da literatura e a experiência pessoal dos pesquisadores. Os dados referentes aos valores do controle metabólico, complicações agudas e crônicas e o tipo de diabetes mellitus não foram incluídos devido aos registros incompletos destas informações nos prontuários, o que poderia comprometer a análise dos resultados.

A coleta de dados foi realizada mediante entrevista, nas visitas domiciliares, utilizando-se os dois instrumentos, previamente elaborados, constituídos com questões dirigidas e estruturadas, e aplicados, respectivamente, aos usuários que fazem a auto-aplicação da insulina (Grupo A) e aos usuários que não realizam a auto-aplicação (Grupo B).

$\mathrm{O}$ instrumento de coleta de dados dirigido ao Grupo A continha dados referentes às condições 
sócio-demográficas e clínicas (sexo, idade, escolaridade, tipo de diabetes e tempo de diagnóstico e de tratamento com a insulina). Ao instrumento de coleta dirigido ao Grupo B, foram acrescidas informações sobre as dificuldades percebidas para a realização da auto-aplicação, o responsável pela aplicação da insulina e participação deste em atividades de educação em saúde e/ou consultas médicas e de enfermagem.

O grau de dependência do usuário em relação ao responsável pela aplicação da insulina também foi observado, sendo considerado como dependente total aquele que necessita de auxílio de outros para a conservação e o armazenamento da insulina e reutilização e descarte de seringas, além do preparo e aplicação do medicamento. Foram classificados como usuários que possuem dependência parcial os que necessitam de auxílio somente nos procedimentos de preparo e aplicação do medicamento.

Após a coleta, os dados obtidos nas entrevistas foram codificados, categorizados e digitados em banco de dados no programa Excel (Microsoft Corp., Estados Unidos), transportados e processados no programa estatístico SPSS versão 15.0.

Foram estudadas as associações, a partir do modelo de regressão logística, da variável resposta dicotômica (auto-aplica e não auto-aplica a insulina) com as seguintes preditoras: sexo, faixa etária, escolaridade, tempo de diagnóstico e de tratamento com insulina. Considerando os objetivos desta pesquisa, foi empregado somente o método de entrada forçada com todos os preditores simultaneamente. Adotou-se um erro de primeira espécie de $5 \%$.

O desenvolvimento deste estudo foi aprovado pela coordenação da Estratégia Saúde da Família do município onde o estudo foi realizado e pelo Comitê de Ética e Pesquisa com Seres Humanos da Universidade Federal do Triângulo Mineiro (protocolo 527).

\section{Resultados}

Dos 269 usuários participantes do estudo, 169 $(62,8 \%)$ fazem a auto-aplicação de insulina e 100 $(37,2 \%)$ não. Os dados referentes às características sócio-demográficas dos dois grupos distintos (A e B) estão apresentados na Tabela 1.

Os dados, apresentados na Tabela 1, apontam maior freqüência de usuários do sexo feminino, tanto no Grupo A (71\%) quanto no B (72\%).

Com relação à idade, a maior freqüência encontra-se na faixa etária maior ou igual a 60 anos para os dois grupos (Tabela 1). No Grupo A, a me-

Tabela 1

Distribuição numérica e percentual dos grupos A e B acompanhados pela Estratégia Saúde da Família, segundo sexo, faixa etária e escolaridade, 2007

\begin{tabular}{|c|c|c|c|c|c|c|}
\hline \multirow[t]{2}{*}{$\begin{array}{l}\text { Variáveis } \\
\text { sócio-demográficas }\end{array}$} & \multicolumn{2}{|c|}{$\begin{array}{l}\text { Auto-aplica insulina } \\
\text { Grupo A (N = 169) }\end{array}$} & \multicolumn{2}{|c|}{$\begin{array}{c}\text { Não auto-aplica insulina } \\
\text { Grupo } B(N=100)\end{array}$} & \multicolumn{2}{|c|}{ Total } \\
\hline & $\mathrm{n}$ & $\%$ & $\mathrm{n}$ & $\%$ & $\mathrm{n}$ & $\%$ \\
\hline \multicolumn{7}{|l|}{ Sexo * } \\
\hline Feminino & 120 & 71,0 & 72 & 72,0 & 192 & 71,4 \\
\hline Masculino & 49 & 29,0 & 28 & 28,0 & 77 & 28,6 \\
\hline Total & 169 & 100,0 & 100 & 100,0 & - & - \\
\hline \multicolumn{7}{|l|}{ Faixa etária (anos) ** } \\
\hline$<60$ & 76 & 45,0 & 29 & 29,0 & 105 & 39,0 \\
\hline$\geq 60$ & 93 & 55,0 & 71 & 71,0 & 164 & 61,0 \\
\hline Total & 169 & 100,0 & 100 & 100,0 & - & - \\
\hline \multicolumn{7}{|l|}{ Escolaridade (anos) } \\
\hline Sem escolaridade & 38 & 22,5 & 54 & 54,0 & 92 & 34,2 \\
\hline $1-8 * \star \star$ & 108 & 63,9 & 40 & 40,0 & 148 & 55,0 \\
\hline$>9 \#$ & 23 & 5,3 & 6 & 6,0 & 29 & 10,8 \\
\hline Total & 169 & 100,0 & 100 & 100,0 & - & - \\
\hline
\end{tabular}

${ }^{*} \mathrm{p}=0,96 ; \mathrm{RC}=1,02 ; \mathrm{IC} 95 \%: 0,55-1,82 ;$

** $p=0,198 ; R C=1,48 ; 1 C 95 \%: 0,82-2,68$;

*** $p=0,003 ; R C=0,21 ;$ IC $95 \%: 0,76-2,59$;

$\# p=0,662 ; R C=0,80 ;$ IC95\%: 0,29-2,17. 
diana foi de 61, valor mínimo de 18 e máximo de 99 anos de idade; já em relação ao Grupo B, a mediana foi de 67, valor mínimo de 18 e máximo de 93 anos de idade.

Quanto à escolaridade, verifica-se uma pequena freqüência de usuários com mais de nove anos de estudos nos dois grupos (Tabela 1). É importante destacar que 94\% dos usuários do Grupo B têm menos de oito anos de estudo.

Em relação ao tipo de diabetes mellitus, verificou-se na população do estudo $(\mathrm{N}=269)$ que $122(45,4 \%)$ usuários não souberam informar. Dos que souberam informar, no Grupo A, 16 (17\%) referiram ter diabetes mellitus 1 e 78 (83\%) diabetes mellitus 2, enquanto que no Grupo B, $6(11,3 \%)$ referiram ter o diabetes mellitus 1 e 47 $(88,7 \%)$ diabetes mellitus 2 . O desconhecimento referido do tipo de diabetes mellitus e a inconsistência destes dados nos prontuários limitaram a comparação desta variável entre os grupos.

No que se refere ao tempo de diagnóstico do diabetes mellitus, a mediana encontrada foi de 12 anos, tendo o valor mínimo de 1 e máximo de 40 anos, nos grupos A e B. Já em relação ao tempo de insulina, a mediana foi de 6 anos, tendo o valor mínimo de 1 e máximo de 30 anos no Grupo A, enquanto que no Grupo B, a mediana foi de 5, o valor mínimo de 1 e o máximo de 25 anos.

Ao comparar os grupos A e B em relação às variáveis sócio-demográficas e clínicas, por meio da análise bivariada (qui-quadrado), foi verificada diferença estatisticamente significante para as variáveis faixa etária $(p=0,009)$ e escolaridade ( $p<0,001$ ), ou seja, os usuários do Grupo B eram mais velhos e com menor escolaridade.

No entanto, quando as variáveis sócio-demográficas (Tabela 1) e clínicas foram submetidas à análise multivariável, usando-se o modelo de regressão logística, a única preditora estatisticamente significante foi a escolaridade $(p=0,003)$, ou seja, foi observado que quanto menores forem os anos de estudo, maiores serão as chances para os usuários não auto-aplicarem a insulina.

O nível de significância (p) encontrado na análise multivariada entre os grupos, para as variáveis clínicas, foi 0,32 para o tempo de diagnóstico e 0,65 para o tempo de tratamento com insulina.

Na Tabela 2, em relação às pessoas mencionadas como responsáveis pela aplicação da in-

Tabela 2

Distribuição numérica e percentual dos usuários do Grupo B acompanhados pela Estratégia de Saúde da Família, segundo o responsável pela aplicação de insulina e os fatores que dificultam a aplicação, 2007.

\begin{tabular}{|c|c|c|}
\hline Não fazem a auto-aplicação de insulina - Grupo B $(N=100)$ & $\mathbf{n}$ & $\%$ \\
\hline \multicolumn{3}{|l|}{ Dificuldades percebidas para a auto-aplicação da insulina } \\
\hline Medo/Insegurança & 37 & 37,0 \\
\hline Dificuldades visuais & 28 & 28,0 \\
\hline Dificuldades motoras & 17 & 17,0 \\
\hline Dificuldades cognitivas & 6 & 6,0 \\
\hline Acamado & 4 & 4,0 \\
\hline Outros & 8 & 8,0 \\
\hline Total & 100 & 100,0 \\
\hline \multicolumn{3}{|l|}{ Responsável pela aplicação da insulina no domicílio } \\
\hline Familiares & 75 & 75,0 \\
\hline Profissionais & 19 & 19,0 \\
\hline Outros & 6 & 6,0 \\
\hline Total & 100 & 100,0 \\
\hline \multirow{2}{*}{\multicolumn{3}{|c|}{$\begin{array}{l}\text { Participação/acompanhamento do responsável pela aplicação em } \\
\text { atividades de educação e/ou consultas }\end{array}$}} \\
\hline & & \\
\hline $\operatorname{Sim}$ & 50 & 50,0 \\
\hline Não & 50 & 50,0 \\
\hline Total & 100 & 100,0 \\
\hline \multicolumn{3}{|l|}{ Grau de dependência do usuário } \\
\hline Dependência total & 90 & 90,0 \\
\hline Dependência parcial & 10 & 10,0 \\
\hline Total & 100 & 100,0 \\
\hline
\end{tabular}


sulina, destacou-se o predomínio dos familiares (75\%), sendo que 37 (37\%) usuários recebem auxílio dos filhos, 23 (23\%) do cônjuge, 6 (6\%) do pai/mãe, 6 (6\%) do neto, 2 (2\%) do irmão e 1 (1\%) do genro. Quanto aos profissionais responsáveis pela aplicação da insulina tem-se o técnico de enfermagem (13\%) e o profissional da farmácia (6\%). Os outros foram representados por vizinhos (3\%) e amigos (3\%).

Observa-se, também, que 50 (50\%) pessoas responsáveis pela aplicação da insulina participam e acompanham os usuários em atividades de educação em saúde, consultas médicas ou de enfermagem. Quando indagado aos usuários sobre as dificuldades para a auto-aplicação da insulina, verificou-se que 55 (55\%) referiram apresentar algum tipo de alteração funcional e 37 (37\%) medo da dor e de cometer erros na aplicação do medicamento. Os outros foram categorizados como comodidade ou desânimo (8\%).

Quanto ao grau de dependência, 90 (90\%) usuários referiram necessitar de total colaboração de outros em todo o processo da administração da insulina no domicílio, não somente no preparo como também na aplicação (Tabela 2).

\section{Discussão}

Ao comparar os grupos A e B, verificou-se que não houve associação do sexo para a adesão à auto-aplicação de insulina, porém estudos mostram as relações de poder entre os gêneros, determinando interferências no modo de autocuidado dos sujeitos e destacando, geralmente, as mulheres como responsáveis por uma maior participação no autocuidado 14,15.

Em relação à idade não foi identificada diferença estatisticamente significativa entre os grupos, o que pode ser devido a um viés da amostra. No entanto, ao propor intervenções de saúde, deve-se considerar essa variável, uma vez que as limitações funcionais podem prejudicar o desenvolvimento de habilidades para o autocuidado, dificultar a autonomia e, conseqüentemente, a adesão ao tratamento 16 .

Desse modo, é fundamental que os profissionais da Estratégia Saúde da Família compreendam o processo de envelhecimento, de forma a direcionarem seus esforços para ações educativas que considerem tais limitações, pois as diferentes fases da vida apresentam grande influência na motivação, na adesão e na habilidade de aprendizado para o manejo do diabetes mellitus 17.

A baixa escolaridade encontrada (menos de oito anos de estudo), principalmente em relação aos usuários que não auto-aplicam a insulina $(85,7 \%)$, foi a única preditora estatisticamente significante entre os dois grupos $(p=0,003)$, ou seja, parece haver uma associação entre a baixa escolaridade com a não auto-aplicação de insulina. Estudos mostram que a baixa escolaridade pode dificultar o acesso às informações 13,18,19 e, desta forma, prejudicar o desempenho do autocuidado de forma segura 19 .

Outros estudos confirmaram que as pessoas com maior nível educacional aderem mais ao tratamento e apresentam melhor controle metabólico, assim como apontaram a escolaridade com um fator facilitador para a adesão ao tratamento $19,20,21$.

Neste estudo não foi encontrada associação do tempo de diagnóstico $(\mathrm{p}=0,32)$ e de tratamento com a insulina ( $p=0,65)$ com a adesão à auto-aplicação, apesar de estudos terem demonstrado a relação do tempo de doença 1,22 e, talvez, do tempo de uso da insulina 23 com a evolução da retinopatia, complicação esta que pode interferir em tal adesão 24 .

Outros estudos mostraram que o tempo de doença interfere nas habilidades para o desenvolvimento correto da técnica de auto-aplicação, assim como desmotiva o usuário, e eles passam a não dar importância para o tratamento 9,16,19.

Desse modo, compete ao profissional da Estratégia Saúde da Família identificar os fatores facilitadores e dificultadores à adesão para a auto-aplicação da insulina, para, assim, planejar adequadamente os métodos a serem utilizados para o aprendizado desses usuários, pois os fatores sócio-demográficos e clínicos têm relação com a incapacidade funcional da doença, podendo, desta maneira, interferir nas habilidades individuais para a adesão ao autocuidado 19 .

Porém, na vigência de déficits motores, visuais e neurológicos, muitas vezes será difícil a auto-aplicação correta e segura da insulina 24,25. Entre as dificuldades percebidas pelo usuário para não aderirem à auto-aplicação, observou-se que $28 \%$ referiram déficit visual; $17 \%$ impossibilidade motora, como tremor e imobilidade articular; $6 \%$ déficits cognitivos; e $4 \%$ estão acamados. Esses achados podem estar relacionados às características da população do estudo: diabéticos com média do tempo de diagnóstico de 12 anos e maioria idosos.

As dificuldades motoras e visuais advindas das complicações crônicas do diabetes mellitus, assim como do processo de senilidade, prejudicam a capacidade de desempenho das habilidades de auto-aplicação de insulina, automonitorização da glicose e inspeção da pele e dos pés, interferindo direta ou indiretamente na adesão ao autocuidado 24,25 . 
Alguns recursos podem auxiliar aqueles que apresentam dificuldades em visualizar as escalas de graduação impressas na seringa e em manusear este instrumental, tais como: indicação ou adequação de óculos, ampliadores de escala, seringas com menor capacidade volumétrica e caneta injetora de insulina 24.

Por outro lado, foi verificado que 45 (45\%) usuários têm potencial para assegurar a capacidade de decisão para a manutenção do seu estado de saúde, pelo fato de não apresentarem limitações físicas e/ou cognitivas. Por isso, neste estudo acredita-se que a porcentagem ideal de usuários que auto-aplicam a insulina poderia ser de $80 \%$, em vez de $62,8 \%$.

No entanto, sabe-se também que, além das limitações físicas e cognitivas, os fatores psicossociais e comportamentais interferem na mudança de comportamento para o autocuidado 19,26,27. Alguns estudos descrevem a falta de conhecimento, a desmotivação e o medo da falha pessoal e da dor da aplicação da insulina como uma das barreiras mais comuns para adesão ao tratamento $19,26,27,28$, confirmando os achados deste estudo.

Entre esses fatores, a literatura traz os modos de enfrentamento frente à doença crônica 19,26,27, os quais deveriam ser identificados pelos profissionais e reconhecidos pelos usuários com diabetes mellitus, para otimizar os recursos internos e externos mediante as demandas da doença e cuidados, com a finalidade de adaptação 27.

Por isso, acredita-se, então, que desencorajando os modos de enfrentamento ineficazes e estimulando os que promovem adaptação, conseqüentemente será favorecida a adesão ao tratamento 27 . O processo educativo poderá subsidiar uma das opções para enfrentar as demandas da doença, quando o usuário utiliza a busca de informações como uma forma de enfrentamento 29. A Estratégia Saúde da Família, pelas suas características de proximidade e envolvimento com os usuários, parece favorecer esse tipo de intervenção.

Por essas razões, os profissionais de saúde da Estratégia Saúde da Família deverão estabelecer intervenções sustentadas nas necessidades físicas e psicossociais 27 , porém quando detectadas dificuldades para atingir o objetivo de estimular a auto-aplicação da insulina, o profissional de saúde deverá envolver a família na responsabilidade deste procedimento.

A família, devido às suas características próprias de proximidade e convivência, tem melhores condições de acompanhar os processos de saúde e doença de seus membros. Assim, compete à família não apenas seguir as orientações dos profissionais, mas reconhecer e assumir a responsabilidade pela saúde de seus familiares. Precisa ter dúvidas esclarecidas e opinião considerada, além da participação incentivada no processo do cuidar 30 .

Neste estudo a família teve uma representatividade expressiva no auxílio do processo de administração de insulina entre os usuários que não realizam a auto-aplicação (75\%). No entanto, destaca-se também a participação de profissionais da saúde (18\%), em especial o técnico de enfermagem.

O fato exposto reforça a necessidade de buscar alternativas de incentivo à auto-aplicação e formas de engajar as famílias nos cuidados diários, face às dificuldades de locomoção de seus membros de suas casas até as unidades da Estratégia Saúde da Família, alterando suas atividades da vida diária 11 .

Quanto à participação dos familiares nas atividades educativas e consultas médicas e de enfermagem (50\%), o percentual encontrado pode representar obstáculos para o sucesso do tratamento, principalmente para aqueles que necessitam de auxílio para o autocuidado, por serem em sua maioria, idosos e apresentarem baixa escolaridade. As limitações funcionais e de aprendizagem sustentam a necessidade do acompanhamento do familiar ou cuidador para reforçar as orientações para o autocuidado 19,30,31,32.

Nesse aspecto, o profissional de saúde da Estratégia Saúde da Família deverá conhecer como cada membro da família cuida e identifica suas potencialidades, suas dificuldades e seus esforços para, então, verificar qual membro tem condições para receber e partilhar responsabilidades e, com ele, pensar e implementar a melhor assistência possível 32 .

Vale a pena destacar que a construção do saber acontece quando existe a mútua colaboração entre quem ensina e quem aprende 33 , deste modo o não-envolvimento freqüente do responsável pela aplicação da insulina nas atividades educativas poderá comprometer a realização do procedimento de forma correta e segura 30,34.

Em relação ao grau de dependência, $90 \%$ dos usuários entrevistados relataram que são dependentes em todo o processo da administração da insulina e não somente na aplicação.

O termo dependência tem sido considerado como estado, e a sua existência está relacionada a algo ou alguém que depende de "algo" ou de "alguém” para realizar determinada tarefa. Esse termo liga-se a um conceito fundamental na prática geriátrica: a "fragilidade", condição observada nos usuários com mais de 85 anos ou em crianças e naqueles adultos mais jovens que apresentam uma combinação de doenças e/ou limitações funcionais 35 . 
Apesar de a maioria dos usuários que não auto-aplicam a insulina possuírem mais de 60 anos $(71 \%)$ e $55 \%$ relatarem que apresentam limitações que dificultam a realização de tal procedimento, é necessário, inicialmente, avaliar se a autonomia para o exercício das atividades da vida diária destes usuários não está sendo retirada pela família, pelos profissionais de saúde ou até pelo próprio usuário, que associam alguma perda física à perda cognitiva e à incapacidade decisória, o que, em muitas ocasiões, não ocorre 32 .

Sabe-se que, a dependência não é um estado permanente, é um processo dinâmico cuja evolução pode se modificar e até ser prevenida ou reduzida se houver assistência adequada 32,36 . Trata-se de um processo fundamentalmente educativo 36 .

É importante também destacar que mudar os comportamentos frente às práticas de saúde é uma tarefa difícil, pois exige dos profissionais de saúde: tempo, recursos e capacitação pedagógica para a busca de alternativas metodológicas com a finalidade de sensibilizar o grupo-alvo para o autocuidado, assim como apoiar a família no cuidado domiciliar 37 .

Diante do exposto, a Estratégia Saúde da Família é um modelo de atenção à saúde eficiente para fazer face a esse desafio. As diretrizes que norteiam a Estratégia Saúde da Família permitem maior aproximação e interação dos profissionais e usuários, facilitando o estabelecimento da confiança, comunicação e vínculo, de maneira tal que ambos encontrem melhores soluções que atendam às necessidades dos usuários e da comunidade 38 .

Nessa perspectiva, a Estratégia Saúde da Família também traz a discussão sobre os referenciais teóricos e metodológicos mais adequados para a transformação da prática educativa, contrária à abordagem tradicional centrada na mudança de comportamento individual, destacando o direito à saúde como eixo norteador e a capacidade de escolha dos usuários, assim como o aumento do controle dos determinantes da saúde 38

Percebe-se, desse modo, que há uma necessidade dos profissionais da Estratégia Saúde da Família em adequar e reorganizar a assistência, potencializando e capacitando a população para o autocuidado, identificando seu nível de dependência, suas capacidades presentes e, sobretudo, seu potencial remanescente que pode não estar sendo reconhecido, sequer, pelo próprio usuário e muito menos pelas pessoas que o cercam 32 .

\section{Conclusão}

Os usuários devem ser estimulados a realizar o autocuidado, incluindo a auto-aplicação da insulina, a não ser, em alguns casos, na vigência de limitações físicas e cognitivas, como também, sabe-se que as intervenções em saúde mostramse prejudicadas quando estas não consideram as diferenças/limitações individuais e a relação das variáveis sócio-demográficas e clínicas na incapacidade funcional da doença.

Nesse sentido, foi observado, no presente estudo, que $45 \%$ dos usuários não referiram limitações físicas ou cognitivas que os impedissem de realizar a auto-aplicação da insulina e que a escolaridade foi a única variável sócio-demográfica que obteve diferença estatística entre os dois grupos para a adesão à auto-aplicação da insulina.

O presente estudo apresentou limitações devido aos registros incompletos nos prontuários, que poderão ser investigadas em estudos futuros, tais como, tipo de diabetes mellitus, resultado dos exames laboratoriais e complicações advindas do diabetes mellitus. Seria interessante analisar a associação dos resultados encontrados ao controle glicêmico, verificar a associação do tipo de diabetes mellitus entre os grupos, como também aprofundar a análise das variáveis estudadas.

Desse modo, os dados encontrados neste estudo reforçam a necessidade de elaborar estratégias de educação em saúde que considerem as limitações de cada usuário para estimulá-los a realizarem a auto-aplicação da insulina, como também envolver maior participação dos familiares neste processo.

Espera-se, então, que as compreensões dessas variáveis juntamente com a Estratégia Saúde da Família permitirão propor ações centradas nas reais necessidades da clientela adscrita, estimulando-a para o autocuidado, em específico para a auto-aplicação da insulina. 


\section{Resumo}

Estudo seccional que objetivou comparar dois grupos de usuários com diabetes mellitus acompanhados pela Estratégia Saúde da Família e distinguidos pela auto-aplicação ou não de insulina, em relação às variáveis sócio-demográficas, às dificuldades percebidas e ao responsável pela auto-aplicação. Participaram 269 usuários selecionados por meio de uma amostragem aleatória simples, em 37 unidades da Estratégia Saúde da Família, da área urbana de um município do Estado de Minas Gerais, Brasil. O grupo que faz auto-aplicação foi composto por 169 (62,8\%) usuários e o que não faz, por 100 (37,2\%). Ao comparar os grupos, a variável escolaridade foi estatisticamente significativa; $45 \%$ dos que não auto-aplicam a insulina referiram ausência de dificuldades físicas ou cognitivas que impedissem a realização deste procedimento, demonstrando potencial para a adesão; $90 \%$ referiram necessitar de auxílio em todo o processo de administração de insulina no domicílio e destes, $75 \%$ informaram receber auxílio da família. A Estratégia Saúde da Família favorece o desenvolvimento de intervenções centradas nas necessidades da clientela adscrita, estimulando-a para o autocuidado, e os resultados do presente estudo poderão contribuir com o planejamento destas intervenções.

Diabetes Mellitus; Insulina; Autocuidado; Enfermagem; Saúde da Família

\section{Referências}

1. Engelgau ME, Nayaran KM, Herman WH. Screening for type 2 diabetes. Diabetes Care 2000; 23:1563-80.

2. American Diabetes Association. Diagnosis and classification of diabetes mellitus. Diabetes Care 2004; 27 Suppl 1:S5-10.

3. The effect of intensive treatment of diabetes on the development and progression of long-term complications in insulin-dependent diabetes mellitus. The Diabetes Control and Complications Trial Research Group. N Engl J Med 1993; 329:977-86.

4. Sociedade Brasileira de Diabetes. Aplicação de insulina. In: Sociedade Brasileira de Diabetes, organizador. Tratamento e acompanhamento do diabetes mellitus. Diretrizes da Sociedade Brasileira de Diabetes. Rio de Janeiro: Sociedade Brasileira de Diabetes; 2006. p. 122-5.

5. Robertson KE, Glazer B, Campbell K. The latest developments in insulin injection devices. Diabetes Educ 2000; 26:135-52.

6. Camata DG. Complicações locais na pele relacionadas à aplicação de insulina. Rev Latinoam Enferm 2003; 11:119-22.

\section{Colaboradores}

T. S. G. Stacciarini participou da revisão da literatura, elaboração da metodologia, análise dos resultados e redação do artigo final. V. J. Haas colaborou com a análise dos resultados e redação do artigo final. A. E. Pace contribuiu na revisão da literatura, elaboração da metodologia e revisão do artigo final.
7. Castro ADRV. Reutilização de seringas descartáveis para aplicação de insulina: uma prática comum no domicílio de pacientes com diabetes mellitus [Dissertação de Mestrado]. São Paulo: Universidade de São Paulo; 2005.

8. American Diabetes Association. Insulin administration. Diabetes Care 2004; 27 Suppl 1:S106-9.

9. Mendes IAC, Trevisan MA. Sobre a negligência do paciente diabético insulino-dependente. Enfermagem Atual 1981; 3:17-21.

10. Rossi VEC, Pace AE. Reutilização de seringa de insulina em uma população de diabéticos da Cidade de Passos - Minas Gerais. Nursing (São Paulo) 2001; 4:30-4

11. Silveira VMF, Menezes AMP, Post CLA. Uma amostra de pacientes com diabetes tipo 1 no sul do Brasil. Arq Bras Endocrinol Metab 2001; 45:433-40.

12. Assunção MCF, Santos IS, Dias-da-Costa JS. Avaliação do processo de atenção médica: adequação do tratamento de pacientes com diabetes mellitus, Pelotas, Rio Grande do Sul, Brasil. Cad Saúde Pública 2002; 18:205-11. 
13. Alves VS. Um modelo de educação em saúde para o Programa Saúde da Família: pela integridade da atenção e reorientação do modelo assistencial. Interface Comun Saúde Educ 2005; 9:39-52.

14. Gomes R, Nascimento EF, Araújo FC. Por que os homens buscam menos os serviços de saúde do que as mulheres? As explicações de homens com baixa escolaridade e homens com ensino superior. Cad Saúde Pública 2007; 23:565-74.

15. Santos RS. Autocuidado e gênero em diabetes mellitus. Rev Baiana Enferm 2003; 18:39-50.

16. Maia FR, Araújo LR. Aspectos psicológicos e controle glicêmico de um grupo de pacientes com diabetes mellitus tipo 1 em Minas Gerais. Arq Bras Endocrinol Metab 2004; 26:261-6.

17. Maldonato A. Diabetes mellitus: lessons from patient education. Patient Educ Couns 1995; 26:5766.

18. Paiva DCP, Bersusa AAS, Escuder MML. Avaliação da assistência ao paciente com diabetes e/ou hipertensão pelo Programa Saúde da Família do Município de Francisco Morato, São Paulo, Brasil. Cad Saúde Pública 2006; 22:377-85.

19. Delamater AM. Improving patient adherence. Clin Diabet 2006; 24:71-7.

20. Richardson A, Adner N, Nordstrum G. Persons with insulin-dependent diabetes mellitus: acceptance and coping ability. J Adv Nurs 2001; 33:758-63.

21. Peyrot M, Rubin RR, Lauritzen T, Snoek FJ, Matthews DR, Skovlund SG. Psychosocial proble$\mathrm{ms}$ and barriers to improved diabetes management: results of the Cross-National Diabetes Attitudes, Wishes and Needs (DAWN) Study. Diabet Med 2005; 22:1379-85.

22. Spijkerman AM, Dekker JM, Nigpels G, Adriaanse MC, Kostense PJ, Ruwaard D, et al. Microvascular complications at time of diagnosis of type 2 diabetes are similar among diabetic patients detected by targeted screening and patients newly diagnosed in general practice: the hoorn screening study. Diabetes Care 2003; 26:2604-8.

23. Delcourt C, Villatte-Cathelineau B, VauzelleKervroedan F, Papoz L. Clinical correlates of advanced retinopathy in type II diabetic patients: implications for screening. The CODIA-INSERMZeneca Pharma Study Group. J Clin Epidemiol 1996; 49:679-85.

24. Grossi SAA. Educação para o controle do diabetes mellitus. In: Instituto para o Desenvolvimento da Saúde/Universidade de São Paulo/Ministério da Saúde, organizadores. Manual de enfermagem. Brasília: Ministério da Saúde; 2001. p. 155-67.
25. Smeltzer SC, Bare BG. Histórico e tratamento de pacientes com diabetes mellitus. In: Smeltzer SC, Bare BG, editors. Tratado de enfermagem médico-cirúrgica - Brunner \& Suddarth. Rio de Janeiro: Editora Guanabara Koogan; 2005. p. 1215-68.

26. van Dam HA, van der Horst FG, Knoops L, Ryckman RM, Crebolder HF, van den Borne BH. Social support in diabetes: a systematic review of controlled intervention studies. Patient Educ Couns 2005; 59:1-12.

27. Peyrot M, Rubin RR, Siminerio LM. Physician and nurse use of psychosocial strategies in diabetes care: results of the Cross-National Diabetes Attitudes, Wishes and Needs (DAWN) Study. Diabetes Care 2006; 29:1256-62.

28. Peragallo-Dittko V. Removing barriers to insulin therapy. Diabetes Educ 2007; 33 Suppl 3:60S-5S.

29. Gazzinelli MF, Gazzinelli A, Reis DC, Penna CMM. Educação em saúde: conhecimentos, representações sociais e experiências da doença. Cad Saúde Pública 2005; 21:200-6.

30. Funnell MM. Overcoming barriers to the initiation of insulin therapy. Clin Diabet 2007; 25:36-8.

31. Wen LK, Shepherd MD, Parchman ML. Family support, diet and exercise among older Mexican Americans with type 2 diabetes. Diabetes Educ 2004; 30:980-93.

32. Duarte YAO. O processo de envelhecimento e a assistência ao idoso. In: Instituto para o Desenvolvimento da Saúde/Universidade de São Paulo/Ministério da Saúde, organizadores. Manual de enfermagem. Brasília: Ministério da Saúde; 2001. p. 185-96.

33. Freire P. Pedagogia da autonomia: saberes necessários à prática educativa. Rio de Janeiro: Editora Paz \& Terra; 1997.

34. Toljarno M, Hentinen M. Adherence to self-care and glycaemic control among people with insulindependent diabetes. J Adv Nurs 2001; 34:780-6.

35. Hazzard WR, Brerman EL, Blass JP. Principles of geriatric medicine and gerontology. $3^{\text {rd }}$ Ed. New York: McGraw-Hill; 1994.

36. Caldas CP. Envelhecimento com dependência: responsabilidades e demandas da família. Cad Saúde Pública 2003; 19:733-81.

37. Zanetti ML, Mendes IAC, Ribeiro KP. O desafio para o controle domiciliar em crianças e adolescentes diabéticas tipo 1. Rev Latinoam Enferm 2001; 9:32-6.

38. Chiesa AM, Veríssimo MDR. A educação em saúde na prática do PSF. In: Instituto para o Desenvolvimento da Saúde/Universidade de São Paulo/Ministério da Saúde, organizadores. Manual de enfermagem. Brasília: Ministério da Saúde; 2001. p. $34-42$.

Recebido em 15/Mai/2007

Versão final reapresentada em 02/Out/2007 Aprovado em 08/Out/2007 